\title{
Detection and Monitoring Intra/Inter Crosstalk in Optical Network on Chip
}

\author{
Ahmed Jedidi \\ College of Engineering, Ahlia University, Bahrain \\ CES Laboratory, National Engineering School of Sfax, University of Sfax, Tunisia
}

\begin{tabular}{l} 
Article Info \\
\hline Article history: \\
Received Mar 30, 2018 \\
Revised Jun 18, 2018 \\
Accepted Jul 29, 2018 \\
\hline
\end{tabular}

Keyword:

Inter/intra crosstalk MPSoC

$\mathrm{ONoC}$

Optical communciation

RTL design

\begin{abstract}
Multiprocessor system-on-chip (MPSoC) has become an attractive solution for improving the performance of single chip in objective to satisfy the performance growing exponentially of the computer applications as multimedia applications. However, the communication between the different processors' cores presents the first challenge front the high performance of MPSoC. Besides, Network on Chip (NoC) is among the most prominent solution for handling the on-chip communication. Besides, NoC potential limited by physical limitation, power consumption, latency and bandwidth in the both case: increasing data exchange or scalability of Multicores. Optical communication offers a wider bandwidth and lower power consumption, based on, a new technology named Optical Network-on-Chip (ONoC) has been introduced in MPSoC. However, ONoC components induce the crosstalk noise in the network on both forms intra/inter crosstalk. This serious problem deteriorates the quality of signals and degrades network performance. As a result, detection and monitoring the impairments becoming a challenge to keep the performance in the ONoC. In this article, we propose a new system to detect and monitor the crosstalk noise in ONoC. Particularly, we present an analytic model of intra/inter crosstalk at the optical devices. Then, we evaluate these impairments in objective to present the motivation to detect and monitor crosstalk in $\mathrm{ONoC}$, in which our system has the capability to detect, to localize, and to monitor the crosstalk noise in the whole network. This system offers high reliability, scalability and efficiency with time running time less than $20 \mathrm{~ms}$.
\end{abstract}

Copyright $\left({ }_{0} 2018\right.$ Institute of Advanced Engineering and Science. All rights reserved.

\section{Corresponding Author:}

Ahmed Jedidi,

Department of Computer Engineering,

Ahlia University,

Bahrain.

Email: ajedidi@ahlia.edu.bh

\section{INTRODUCTION}

The high integration rate of transistor has pushed the semiconductor industry to a shift from the single-core to a multi-core in one chip. Besides, a new paradigm is introduced multiprocessor system one chip (MPSoC). However, MPSoC has to face serious problems in terms of energy consumption, execution time, heat dissipation and data flow. Data transfer between cores in MPSoC became the first challenge to improve these parameters. Hence, Network on Chip (NoC) is emerging as a promising solution providing low energy consumption and high bandwidth to improve the performance of MPSoC. However, according the growing of the performance of computer application NoC become a bottleneck for the scalability and power dissipation in the MPSoC. Indeed, electrical interconnects are not able to boost transmission rates and power dissipation which it makes highly desirable to replace them [1]. 
Optical communication offers a high bandwidth with lower power consumption. In fact, it can achieve bandwidths in the order of terabits per second by exploiting wavelength division multiplexing. Since photonic system has a potential capacity and become attractive to use it to improve communication in the chip [2]. As a result, optical network on chip "ONoC" is presented to replace the traditional NoC.

Microresonators (MRs) and nanophotonic waveguides are the key devices in ONoC. However, these optical devices and waveguides provide transparency (a component is called X-transparent if it forwards incoming signals from input to output without examining the $\mathrm{X}$ aspect of the signal) capabilities which they induce optical vulnerabilities "crosstalk" in ONoC where is does not exist in a conventional NoC. Furthermore, one of the serious problems with transparency is the fact that optical crosstalk is additive, and thus the aggregate effect of crosstalk over a whole ONoC may be more nefarious than a single point of crosstalk [3]. Consequently, optical crosstalk degrades the quality of signals and increases their BER (Bit Error Rate) performance [4]. In fact, both forms of optical crosstalk can arise in ONoC system: intercrosstalk and intra-crosstalk.

Inter-crosstalk arises when the crosstalk signal is at a wavelength sufficiently different from the affected signal's wavelength that the difference is larger than the receiver's electrical bandwidth. Intercrosstalk can also occur through more interactions that are indirect. For example, if one channel affects the gain seen by another channel, as with nonlinearities. In the second place, intra-crosstalk arises when the crosstalk signal is at the same wavelength as that of the affected signal or sufficiently close to it that the difference in wavelengths is within the receiver's electrical bandwidth. Intra-crosstalk arises in transmission links due to reflections [5]-[6].

Crosstalk is one of the major problem arising in $\mathrm{ONoC}$ and it is a barrier of the scalability and the performance for the evolution of the MPSoC. As a result, find a method to detect, localize and monitor crosstalk will be essential. In this paper, we propose a new system to detect and monitor the intra/inter crosstalk in ONoC. Indeed, we present an analytic model for the both forms of crosstalk induced by the ONoC components. Based on this study, we evaluate and point out the importance to detect and monitor crosstalk noise in ONoC. Furthermore, we design and implement a performed hardware system to detect and monitor inter/intra crosstalk in the whole network.

The rest of the paper is organized as follows: The second section will present an extensive overview of the related work in the literature. The third section will describe the inner architecture of the basic devices in $\mathrm{ONoC}$ and the network model used in this work. The fourth section will depicit the analytic model of the crosstalk noise induced in optical components. Mainly, we will describe the crosstalk progress in the different devices and in the whole network. The fifth section will describe the hardware design and implementation of the proposed system of detection and monitor the crosstalk in ONoC. The sixth section will discuss and analyse the fusibility and the different results of our system. Finally, we will conclude and expose our future works.

\section{RELATED WORK}

Optical network on chip provide a promising solution to increase the requirements in ultra-high bandwidth and lower power consumption in the MPSoC. According the progress of technology, nanophotonic waveguide and optical switch are presented in the chip, which they are the key components for ONoC. In the last decade, many researchers focus their works to propose a performed architecture of ONoC in objective to satisfy the different requirements of MPSoC performance. However, the crosstalk noise is one of the most important issues that researchers face in developing optical network on-chip.

In the literature, most of the researchers focus their work on analysis and modeling of crosstalk noises induce by the optical devices in ONoC. Furthermore, many studies isolate their work for modeling and analyzing the crosstalk noise in optical devices separately as router or waveguide. Yiyuan Xie and al. analyse and optimize cornstalk in 5x5 Hitless Silicon-Based Optical Router [7]. Hence, they analysed crosstalk noise at device level and router level. Based on the detailed analysis, they proposed a general analytical model to study the transmission loss, crosstalk noise, optical signal-to noise ratio (OSNR), and bit error ratio (BER). Indeed, they used the crossing angles of $60^{\circ}$ or $120^{\circ}$ instead of the conventional $90^{\circ}$ crossing angle to design the optical router. Using this method OSNR is improved by about $10 \mathrm{~dB}$ [7].

Fabrizio Gambini and al. proposed a photonic multi-microring NoC, which the theoretical model based on the transfer matrix method, has been validated through experimental results in terms of transmission spectra [8]. Transmissions at $10 \mathrm{~GB} / \mathrm{s}$ have been assessed in terms of BER for both single-wavelength and multi-wavelength configurations. The integrated NoC consists of 8 thermally tuned microrings coupled to a central ring, where the BER measurements show performance up to $10^{-9}$ at $10 \mathrm{~Gb} / \mathrm{s}$ with limited crosstalk and penalty (below $0.5 \mathrm{~dB}$ ) induced by an interfering transmission [8]. 
Besides, many works deal with the crosstalk in the whole ONoC. Mainly, it is how the crosstalk noises effect the performance of the network. Particularly, these studies analyse and model the crosstalk progress in the different parts of the network, and present their effect to BER and SNR of the network.

Mahdi Niksdat and al. systematically study and compare the worst case as well as the average crosstalk noise and SNR in three well known optical interconnect architectures, mesh-based, folded-torusbased, and fat-tree-based ONoCs using WDM [9]. The analytical models for the worst case and the average crosstalk noise and SNR in the different architectures are presented. Furthermore, the proposed analytical models are integrated into a newly developed crosstalk noise and loss analysis platform (CLAP) to analyze the crosstalk noise and SNR in WDM-based ONoCs of any network size using an arbitrary optical router. The analyses' results demonstrate that the crosstalk noise is of critical concern to WDM-based ONoCs: in the worst case, the crosstalk noise power exceeds the signal power in all three WDM-based ONoC architectures, even when the number of processor cores is small, e.g., 64 [10].

Yiyuan Xie and al. analysed and modelled the crosstalk noise, signal-to-noise ratio (SNR), and bit error rate (BER) of optical routers and ONoCs [11]-[12]. The analytical models for crosstalk noise, minimum SNR, and maximum BER in mesh based ONoCs are developed which an automated crosstalk analyser for optical routers is developed. They, find that crosstalk noise significantly limits the scalability of ONoCs. For example, due to crosstalk noise, the maximum BER is $10^{-3}$ on the $8 \times 8$ mesh based ONoC using an optimized crossbar-based optical router. To achieve the BER of $10^{-9}$ for reliable transmissions, the maximum ONoC size is $6 \times 6$.

As we presented above the research works in optical network on chip has limited to analyse and to model crosstalk noise in both optical components and the whole network. However, Edoardo Fusella, Alessandro Cilardo present different study in ONoC which they proposed a new mapping system for ONoC where it consider the direct effect of the crosstalk noise to the architecture of NoC [13]. They propose a class of algorithms that automatically map the application tasks onto a generic mesh-based photonic NoC architecture such that the worst-case crosstalk is minimized. Furthermore, the results show that the crosstalk noise can be significantly reduced by adopting the aware-crosstalk mapping system, thereby allowing higher network scalability, and can exhibit encouraging improvements over application-oblivious architectures [13]-[14].

\section{BASIC OPTICAL DEVICES AND NETWORK MODEL}

Optical communication system offers a high bandwidth and high Quality of Service (QoS). However, optical components in ONoC introduce crosstalk noise. In this section, we describe the progress of crosstalk in the optical device moreover in the whole networks [15].

\subsection{Optical devices model}

Waveguides and micro-resonator (MRs) are the two basic optical elements and extensively used to construct basic optical switching elements and optical routers. In particular, we have two types of optical switches: the parallel switching element (PSE) and the crossing switching element (CSE). These elements are $1 \times 2$ optical switching based to MRs and waveguide crossing [16]-[17].

Figure 1 (a) and (b) present the structure of PSE and CSE respectively in the two states OFF and ON. When the state of MRn is ON, we select to switch the wavelength to form the first waveguide to the second this called node in state ON. However, when the state MRn is OFF we have not any switching operation and in this case, the node is in state OFF [17].

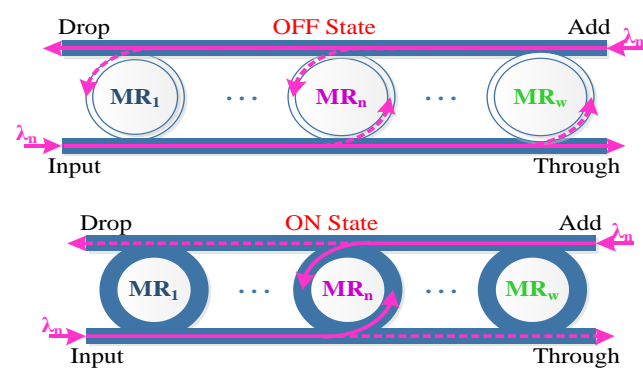

(a)

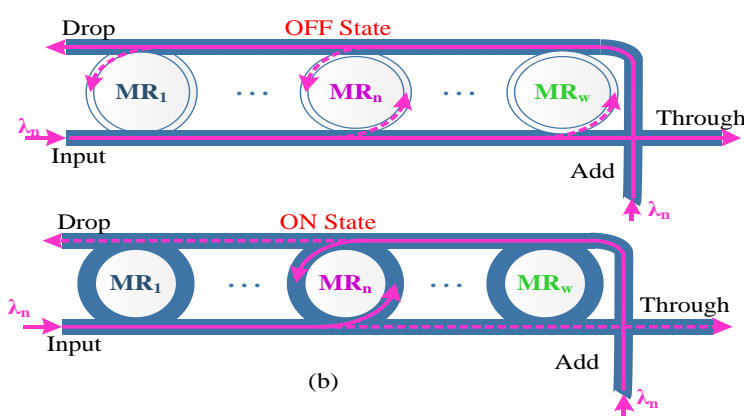

(b)

Figure 1. Basic optical devices in ONoC: (a) PSE, (b) CSE 


\subsection{Network model}

Figure 2 presents an overview of an optical NoC communication between two processors $\mathrm{C} 0$ and $\mathrm{C} 1$. When, the processor $\mathrm{C} 0$ decides to establish connection with the core $\mathrm{C} 1$ an optical signal will be generated with a specific wavelength $\lambda$. Electrical-Optical (E-O) interface on the part of the core C0 starts to convert the electrical signal in optical signal. After, the optical signals passes through the optical on-chip network flowing specific nodes. Finally, the optical signal detected with photodectors in Optical- alectrical (O-E) interface. Thus, optical nodes are the key element in optical NoC and many of them are proposed in different types of network [18]-[19].

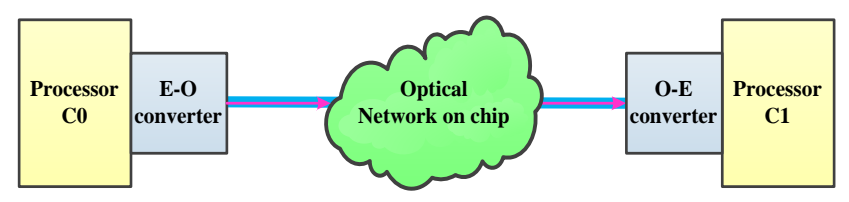

Figure 2. Overview of optical communication via OnoC

To evaluate our system we consider the network model as shown in Figure 3. We assume that the design of the network composed by stages, which the number of stages is direct related with the number of processor cores $\mathrm{N}$. Moreover, the number of stages $\mathrm{P}$ given by the equation 1 , where $\mathrm{N}$ is the total number of processor cores.

$$
P=2 *\left(\frac{\log N}{\log 2}\right)-1
$$

According the number of stages $\mathrm{P}$ in $\mathrm{ONoC}$ the total number of nodes $\mathrm{R}$ is:

$$
R=2 *\left(\sum_{i=1}^{\frac{p}{2}+1} \frac{N}{2}-i\right)-1
$$

The inner architecture of node router composed by particular number $\beta$ of MR according the number of wavelengths $\Lambda$ used in ONoC.

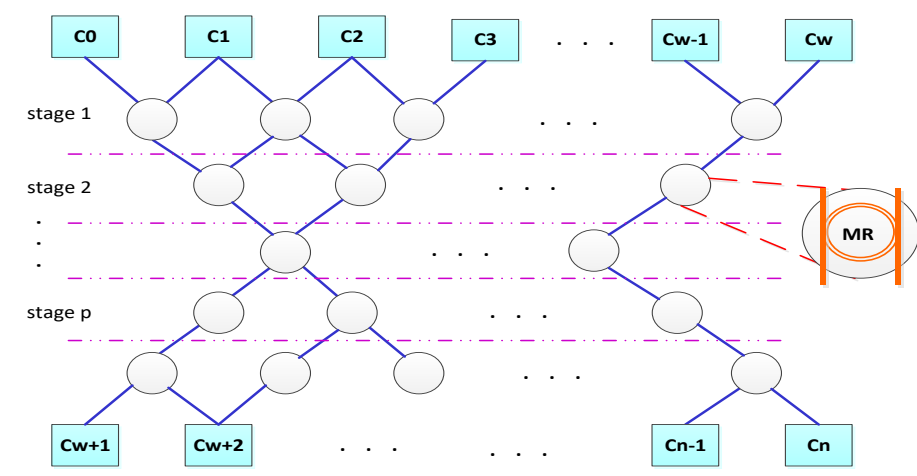

Figure 3. Structure of Optical Network on Chip

\section{CROSSTALK NOISE IN ONOC}

Optical crosstalk is presented in ONoC components and degrades the quality of signals, increasing their BER (Bit Error Rate) performance as they travel through the network [20]. In addition, crosstalk noise increases signal-to-noise ratio (SNR) and affects the quality of service (QoS) of ONoC. In fact, both forms of 
optical crosstalk can arise in ONOC routers: inter-crosstalk and intra-crosstalk [5]. We consider both intrachannel and inter-channel in one crosstalk form and we call it crosstalk noise.

The intrinsic characteristic of photonic devices allows the crosstalk inducing to the optical signal according the wavelengths crossing in the optical devices. We assume, that the rate of the crosstalk noise $\Delta X_{N i}^{\lambda j}$ is induced by the node $\mathrm{i}$ with wavelength $\lambda \mathrm{j}$ and the rate of the crosstalk noise $\Delta Y_{W l}^{\lambda j}$ is induced by the waveguide 1 with wavelength $\lambda \mathrm{j}[21]-[22]$.

We consider the worst case, when each nodes and waveguides of the network immediately induce crosstalk noise (inter/intra crosstalk) corresponding with a specific wavelength $\lambda \mathrm{i}$. The process of communication between cores begin by the initiation from the processor core $\mathrm{M}(\mathrm{CM})$ to connect with the processor core $\mathrm{N}(\mathrm{CN})$. At this time, ONoC defines the route between $\mathrm{CM}$ and $\mathrm{CN}$ and selects a specific wavelength for this request.

This route is composed by a specific number of nodes and waveguides. Therefore, the longest route is composed by $\mathrm{P}$ nodes and $\mathrm{P}+1$ waveguides. However, the shortest route is constituted by 1 node and two waveguides. As mentioned before, the optical components induce directly the crosstalk noise to the optical signal passed through them. Furthermore, we define the total crosstalk noise added to the optical signal $K i$ induced by the different waveguides is:

$$
C r P_{\text {waveguide }}^{K i}=\sum_{l=1}^{K} \sum_{j=1}^{j \nexists i} \Delta Y_{W l}^{\lambda j}
$$

where $\mathrm{k}$ is the number of waveguide which constitute the route between $\mathrm{CM}$ and $\mathrm{CN}$ and $\mathrm{Kj}$ is the wavelength used in ONoC deprive of Ki. Additionally, we present the total crosstalk noise appended to the optical signal $K i$ induced by the different router nodes is:

$$
C r P_{n o d e}^{K i}=\sum_{i=1}^{L} \sum_{j=1}^{j \nexists i} \Delta X_{N i}^{\lambda j}
$$

where $\mathrm{L}$ is the number of router nodes which they are a part of the route between $\mathrm{CM}$ and $\mathrm{CN}$. Then, the total crosstalk noise power in the $\mathrm{CN}$ for the optical signal $K i$ is presented by:

$$
\begin{aligned}
& \operatorname{Cr} P_{N}^{K i}=\operatorname{Cr} P_{\text {waveguide }}^{K i}+\operatorname{Cr} P_{\text {node }}^{K i} \\
& \operatorname{CrP}_{N}^{K i}=\sum_{l=1}^{K} \sum_{j=1}^{j \nexists i} \Delta Y_{W l}^{\lambda j}+\sum_{i=1}^{L} \sum_{j=1}^{j \nexists i} \Delta X_{N i}^{\lambda j}
\end{aligned}
$$

Finally, the SNR of the optical carried on the wavelength $K i$ is:

$$
S N R^{\lambda i}=10 \log \left(\frac{P_{\text {Signal }}^{\lambda i}}{P_{\text {crosstalk }}^{\lambda i}}\right)
$$

where, $P_{\text {Signal }}^{\lambda i}$ is the optical power signal and $P_{\text {crosstalk }}^{\lambda i}$ is the crosstalk noise power.

To point out the dangerous aspect of the crosstalk on the network, we evaluate the SNR and the crosstalk power in ONoC. Besides, we use CLAP tools for the simulation which developed by [9]. We expose the SNR and the power of the crosstalk noise according the size of the network and the number of wavelength used in ONoC. Precisely, we compare between the longest route and the shortest one.

The Figure 4 (a) and (b) present the SNR and Crosstalk power noise according the size of the network for the number of $\Lambda$ equal 8 and 16 respectively. Notice that the crosstalk power noise slightly increase according the size of network, but the SNR decreases.

Furthermore, it is clear that the crosstalk power noise is greater for the longest route than the shortest one, is due to the additive nature of crosstalk noise which it induces each time when they has cross in the route. Consequently, the crosstalk power noise accumulates through the optical route between source core and destination one. We notice that the SNR become so high when the number of wavelength employed in the network are increased. 


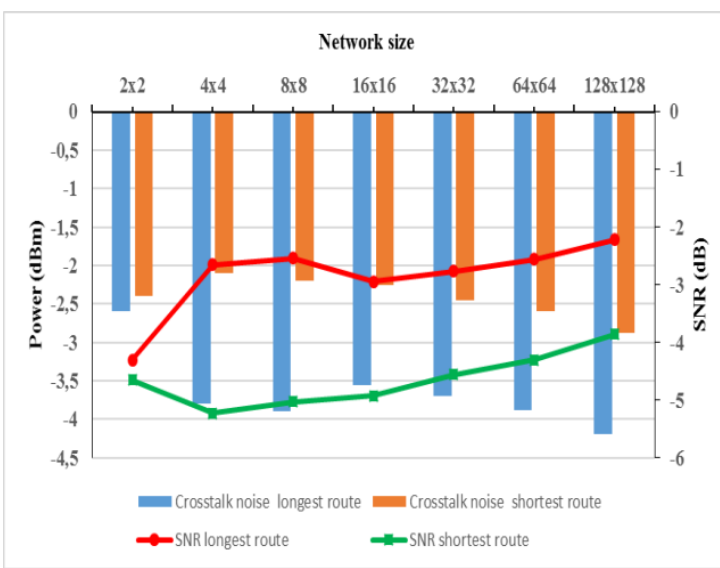

(a) number of $\Lambda=8$

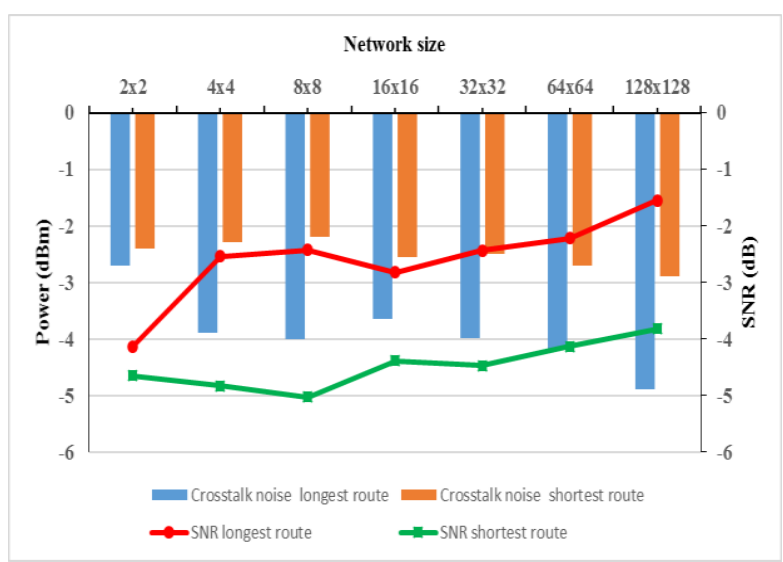

(b) number of $\Lambda=16$

Figure 4. Crosstalk noise power and SNR according the network size

We evaluate the crosstalk power noise and the SNR according the wavelength number employed in the $\mathrm{ONoC}$ as depict in the Figure 5. Particularly, we fixed the network size by $16 \times 16$ cores and we sweep wavelength number from 2 to 32. On account of the increase of the wavelength number, the crosstalk power noise takes an exponential progress. However, the SNR considerably decreases when the number of wavelength increase. Definitely, the presence of high number of optical signals in the same waveguide or optical component causes a direct effect to crosstalk induced in the corresponding wavelength.

Finally, we conclude that the crosstalk noise became critical when we increase the wavelength number or the network size. Accordingly, detect and monitor crosstalk in ONoC became indispensable.

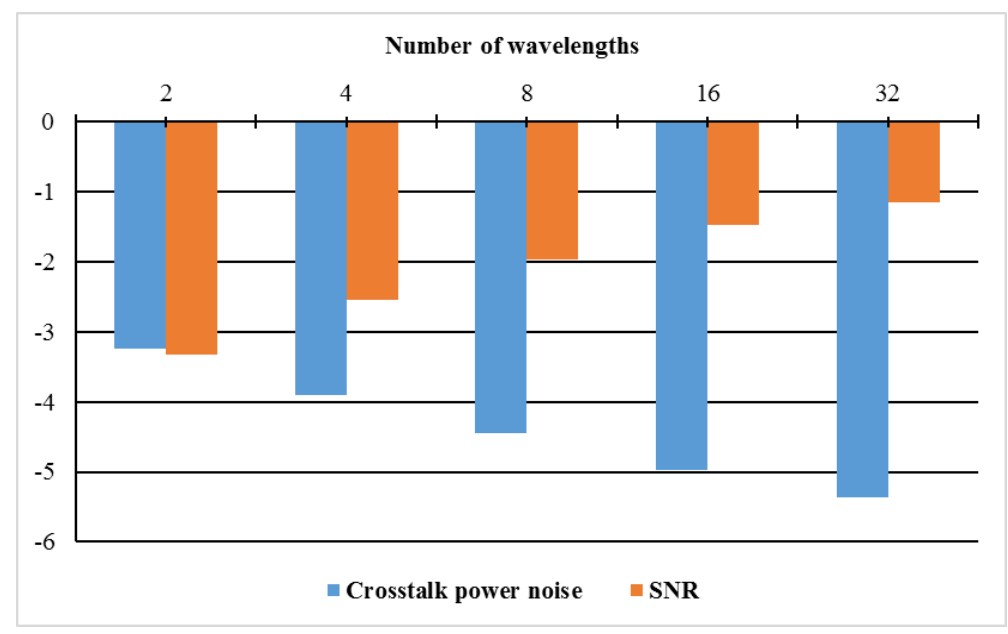

Figure 5. Crosstalk noise and SNR according the number of wavelengths for 16x16 cores

\section{CROSSTALK NOISE DETECTION AND MONITOR SYSTEM}

As mentioned before, crosstalk noise is serious obstacles to develop optical network on chip which the reliability and performance of the MPSoC will be curb. As a Result, find a system that has a capability to detect and monitor the crosstalk noise in $\mathrm{ONoC}$ is essential and vital. Mainly, this system must respect the following requirements:

- Efficiency

- Scalability

- Facility to implement

To detect and monitor crosstalk in $\mathrm{ONoC}$, we propose the Crosstalk Detection and Monitor System (CDMS). The main idea of CDMS is to monitor continuously the different impairments (intra/inter crosstalk) in the whole network. To reach these objectives CDMS is composed by several Crosstalk Detection Bloc 
(CDB) distributed in the network according a special localization system as shown in the Figure 6. Furthermore, CDB is placed between two optical nodes in the same level which CDB splits the various optical signals passed through these optical routers. The process of function of CDB is given by the algorithm 1. First, we split the optical signals from the input/output of the appropriate optical nodes. To realize this operation we use, a photodetector module to convert the optical signals in electrical signals. Second, the different input/output signals passed to CDB which the process of detection and localization of the crosstalk noise launched. Third, a system of crosstalk classification begin to monitor and to classify the different detected impairments according the values of the crosstalk noises. Moreover, the crosstalk noises are classified on 3 types as shown in the Table 1.

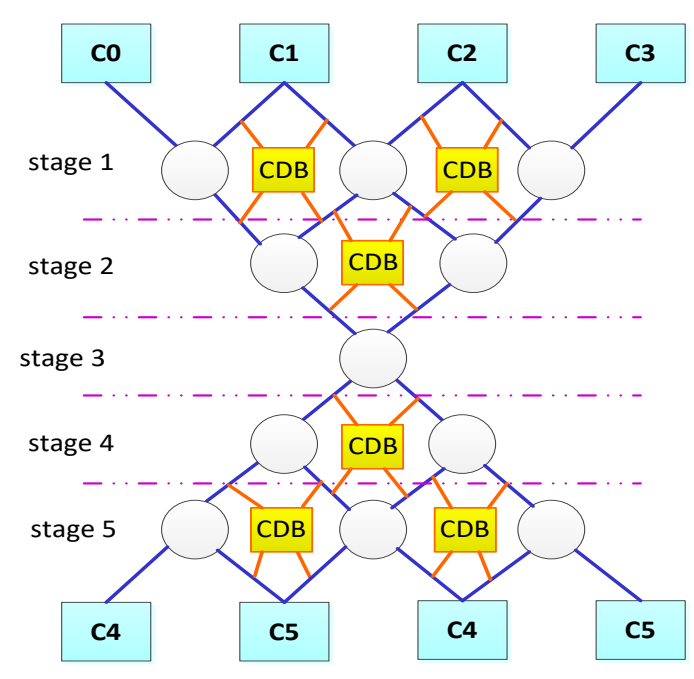

Figure 6. Proposed Crosstalk Detection and Localization System in FONoC

Table 1. Impertment alarms types

\begin{tabular}{|c|c|}
\hline Imperment Alarm & Description \\
\hline No Crosstalk & No detection of crosstalk (healthy signal) \\
\hline Acceptable Crosstalk & $\begin{aligned} \operatorname{Cr} P_{N}^{K i} \leq & 3.2 d b \& \& L_{N}^{K i} \in R_{u p p e r}^{K i}(\text { the upperhalf of the route }) \\
& \left.\operatorname{Cr} P_{N}^{K i}>3.2 d b \forall L_{N}^{\wedge i} \text { (crosstalk location }\right)\end{aligned}$ \\
\hline Dangenrous Crosstalk & $C r P_{N}^{K i} \leq 3.2 d b \& \& L_{N}^{K i} \in R_{\text {lower }}^{K i}$ (the lowerhalf of the route $)$ \\
\hline
\end{tabular}

The CDMS monitor the crosstalk noise by evaluate the status and features of the signal, which the classification of the crosstalk noise is essential. Indeed, when the crosstalk noise power is great than $3.2 \mathrm{db}$ the crosstalk noise is classified as dangerous and we must generate an alarm whatever the localization of the optical component how responsible for it. Besides, this situation named Dangerous crosstalk level. However, when the crosstalk noise power is less than $3.2 \mathrm{db}$, we have two situation:

1. If the optical component how has the responsibility of this impairment is located in the upper-half route, and we classify this crosstalk noise as acceptable. Besides, the acceptable crosstalk level allow to the optical signal reach the destination without alarm but they must be examined.

2. If we detect the crosstalk noise at the lower-half route and alarm is generated and we classify this crosstalk as dangerous. Because, the probability to induce more crosstalk is high and extremely affect the optical signal. 


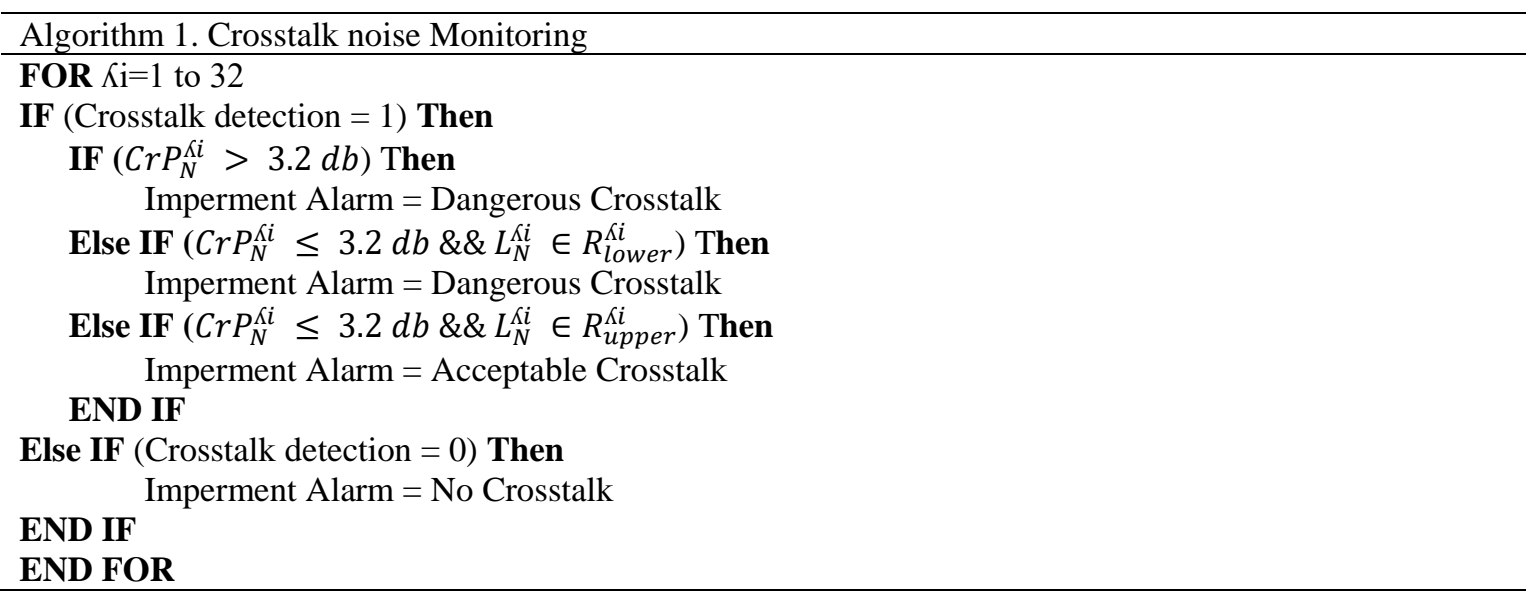

To realize these operations in real-time function we design, simulate and implement the CDMS in RTL. Indeed, the inner architecture of the CDB shown in the Figure 7. Furthermore, CDB composes by several MRi to split the different optical signal $K \mathrm{i}$ which the number of MRi equal to the number of $\Lambda$. Next, we used a photodetector to convert optical signal to electrical one and the number of the photodetectors are the numbers of $\kappa$. For the input signals passed to delay process to synchronize with the output signals. Finally, a system to detect the crosstalk noise presented with the complexity and the cost a directly related with the number of wavelength used.

CDMS is a distributed system use the different informations collected from the CDB devices. We centralize these informations in the CDMS, which we proceed the monitoring of the crosstalk noise in the whole network. Moreover, the CDMS localize and classify the different crosstalk noises in objective to generate the appropriate alarms as depict in the Algorithm 1.

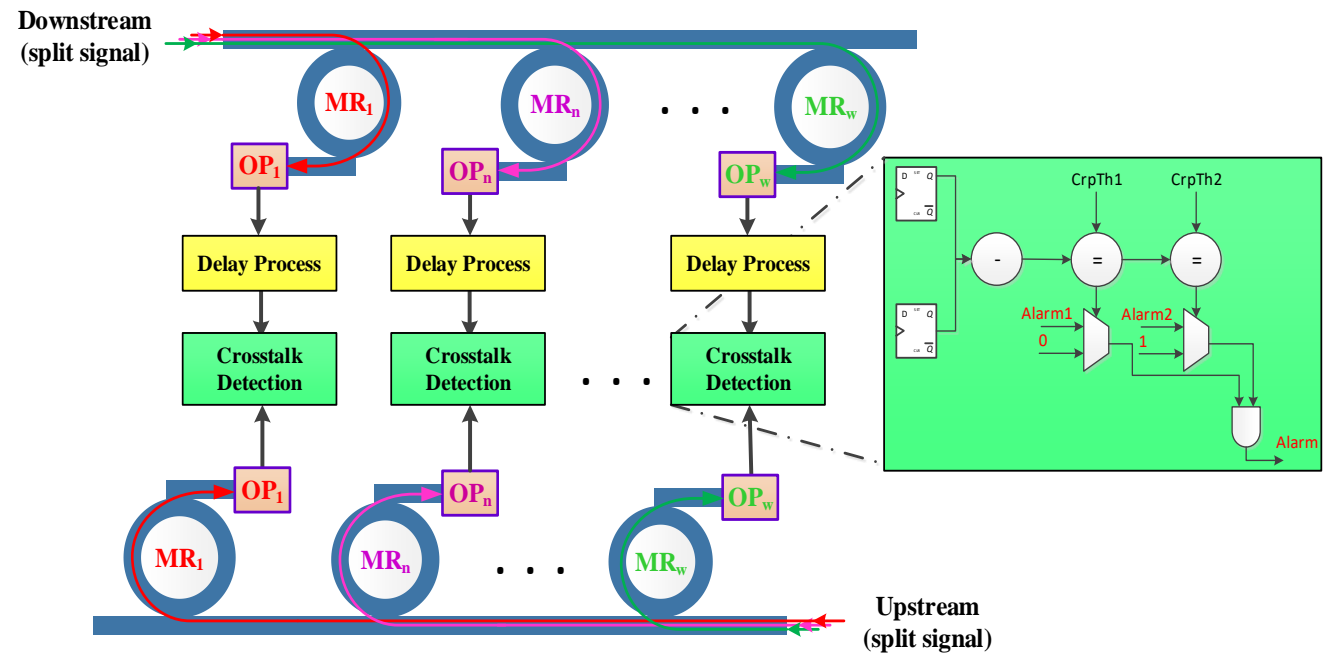

Figure 7. Crosstalk Detection Block

\section{RESULTS AND ANALYSIS}

To discuss the feasibility, the reliability, the scalability and the cost of our system, we simulate, synthesize and implement CDMS in FPGA. Furthermore, we used the STARTER Kit of Xilinx with the different simulation and syntheses tools (Project Navigator of Xilinx and ModelSim). Particularly, we selected SPARTAN-3E for our work [23].

We study the cost, the complexity and the scalability of CDMS according the size of the network and the number of the wavelengths used in ONoC. Besides, these parameters evaluate by the occupation area of the CDMS in the chip as shown in the Figure 8. We notice that the area occupation in the chip is the number of LUTs used on chip. Moreover, the total number of LUTs exponentially increases according the number of processor cores scales. Indeed, this evolution explained by the increase of the number of CDB 
according the size of the network. In addition, we remark that the execution times increases smoothly as function as the number of cores which they reflect the high scalability of CDMS. In particular, the average of the exactions time is around 23 microseconds. As result, CDMS reach a real time function with a high scalability. Indeed, when the network has 2048 processor cores CDMS need less than 4000 LUTs and this value is $0.01 \%$ of the size of the chip and the execution time is less than 25 microseconds. The penalty from the photodetector operation slightly effect the real time function of the CDMS because all devices are implemented in the same chip and the conception of the CDMS boost the real time execution by a high RTL design.

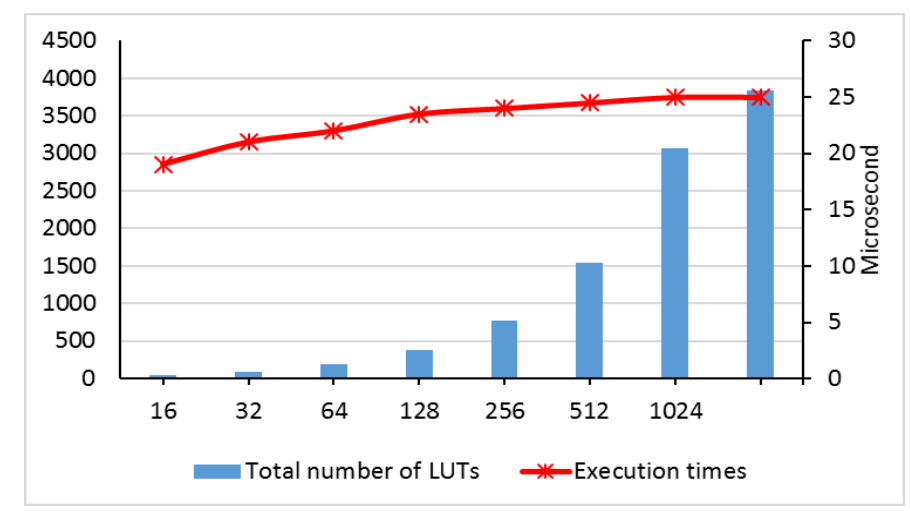

Figure 8. Complexity and scalability of CDLS in chip

To better understanding the cost and the complexity of the CDMS, we explore the cost and the scalability of CDB according the number of the wavelength $K \mathrm{i}$ used in ONoC. The Figure 9 presents the complexity of the CDB define as the rate of the LuTs number over the wavelength number $K$ used in ONoC. Similarly, we present the flow of data process as performance and scalability of CDB. We realize that the complexity of CDB increase significantly when the number of $\Lambda$ is between 2 and 16 then this variation slightly stabilizes. Indeed, this progress of the complexity is due to the reuse of the hardware blocs, which the complexity rate is $64 \%$ for $\Lambda$ equal 16 than it is $62 \%$ for $\Lambda$ equal 32 . Otherwise, we notice that the performance and scalability of CDB are mostly constant as function the number of wavelengths. As a result, the CDB offers a high scalability and performance with an appropriate complexity when the number of wavelengths exceeds 16 .

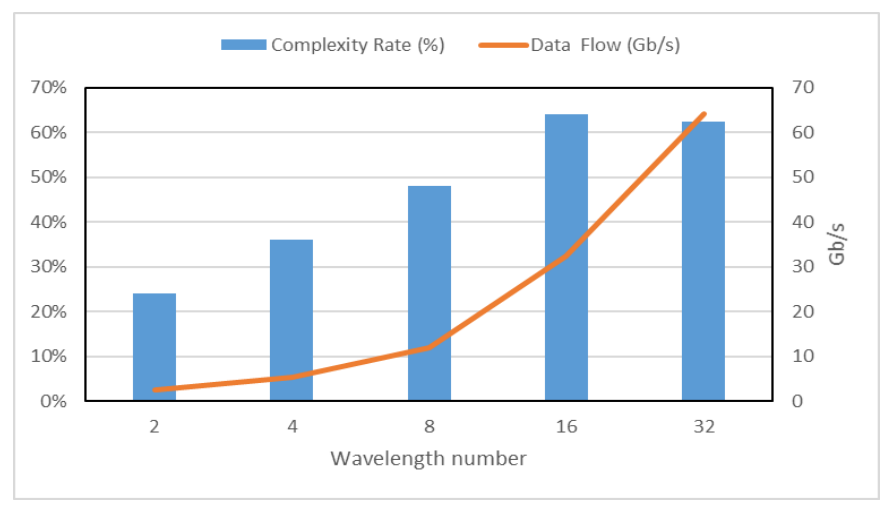

Figure 9. Complexity and Data flow of CDB

\section{CONCLUSION}

The technology growth of transistor integration has now reached its limits. Moreover, this high transistor integration rate has pushed the semiconductor industry to shift from the single-core to a multi-core in one chip (MPSoC). One of the serious problems of the MPSoC is the communications between the 
different processor's cores. In this context, Network on Chip is a promote solution to solve this problem but is limited by the increased number of cores implemented on chip. Optical Network on Chip is a promotional solution that solves the problem of high rate of data exchange between cores with less energy consumption. However, in optical communication ONoC is affected by crosstalk noise, which is a major problem that hinders the achievement and maintenance of high performance. In fact, crosstalk noise deteriorates the quality of signals and hence degrades system's performance. In this paper we proposed a new system to detect and monitor crosstalk noise in $\mathrm{ONoC}$, which the contribution of this work if to offer the first completely system to detect and monitor alarms induced by crosstalk in ONoC. Particularly, we described the distributed architecture and the function of CDMS, also, we focused on the hardware design of CDB. Finally, we implemented and simulated our system to evaluate there performance. The results have demonstrated that, our system offers a high scalability with low rate of occupation in area of the chip as well as a real-time function with 23 microseconds as execution time.

\section{REFERENCES}

[1] E. Bonetto, et al., "Optical technologies can improve the energy efficiency of networks," Proc. 35th Eur. Conf. Opt. Commun., pp. 1-4, 2009.

[2] M. J. Cianchetti, et al., "Phastlane: A rapid transit optical routing network," Proc. 36th Annu. ISCA, pp. 441-450, 2009.

[3] L. Zhou, et al., "A Joint-Coding Scheme with Crosstalk Avoidance in Network on Chip," TELKOMNIKA (Telecommunication Computing Electronics and Control), vol/issue: 11(1), 2013.

[4] M. F. Chatmen, et al., "New Design of Network on Chip Based on Virtual Routers," Indonesian Journal of Electrical Engineering and Computer Science(IJEECS), vol/issue: 2(1), pp. 115-131, 2016.

[5] H. A. M. Harb, et al., "A Study of the Number of Wavelengths Impact in the Optical Burst Switching Core Node," Proceeding of the Electrical Engineering Computer Science and Informatics, Yogyakarta, Indonesia, pp. 664-667, 2017.

[6] A. Jedidi, et al., "Detection and localization of crosstalk in an all-optical network," Journal of optics, vol/issue: 13(1), 2011.

[7] Y. Xie, et al., "Crosstalk Noise Analysis and Optimization in 5x5 Hitless Silicon-Based Optical Router for Optical Networks-on-Chip (ONoC)," Journal of Lightwave Technology, vol/issue: 30(1), 2012.

[8] F. Gambini, et al., "BER evaluation of a low-crosstalk silicon integrated multi-microring network-on-chip," optics express journal, 2015.

[9] M. Nikdast, et al., "Systematic analysis of crosstalk noise in folded-torus based optical networks-on-chip," IEEE Trans. Comput.-Aided Design Integr. Circuits Syst., vol/issue: 33(3), pp. 437-450, 2014.

[10] M. Nikdast, et al., "Crosstalk Noise in WDM-based Optical Networks-on-Chip: a Formal Study and Comparison," IEEE Transactions on Very Large Scale Integration Systems, vol/issue: 23(11), pp. 2552-2565, 2015.

[11] Y. Xie, et al., "Formal worst-case analysis of crosstalk noise in mesh-based optical networks-on-chip," IEEE Trans. Very Large Scale Integr. (VLSI) Syst., vol/issue: 21(10), pp. 1823-1836, 2013.

[12] Y. Xie, et al., "Crosstalk noise and bit error rate analysis for optical network-on-chip," Proc. 47th ACM/IEEE DAC, pp. 657-660, 2010.

[13] E. Fusella and A. Cilardo, "PhoNoCMap: an Application Mapping Tool for Photonic Networks-on-Chip," Design, Automation \& Test in Europe Conference \& Exhibition (DATE), 2016.

[14] E. Fusella and A. Cilardo, "Crosstalk-Aware Automated Mapping for Optical Networks-on-Chip," ACM Trans. Embed. Comput. Syst., vol/issue: 16(1), 2016.

[15] J. Chan, et al., "Physical-layer modeling and system-level design of chip-scale photonic interconnection networks," IEEE Trans. Comput.-Aided Design Integr. Circuits Syst., vol/issue: 30(10), pp. 1507-1520, 2011.

[16] W. Ding, et al., "Compact and low crosstalk waveguide crossing using impedance matched metamaterial," Appl. Phys. Lett., vol/issue: 96(11), pp. 111114-1-111114-3, 2010.

[17] Q. Li, et al., "Design and demonstration of compact, wide bandwidth coupled-resonator filters on a silicon-oninsulator platform," Opt. Exp., vol/issue: 17(4), pp. 2247-2254, 2009.

[18] D. Nikolova, et al., "Scaling silicon photonic switch fabrics for data center interconnection networks," Opt. Express, vol/issue: 23(2), pp. 1159-1175, 2015.

[19] M. Petracca, et al., "Design Exploration of Optical Interconnection Networks for Chip Multiprocessors," HOTI, 2008.

[20] L. H. K. Duong, et al., "A case study of signal-to-noise ratio in ring based optical networks-on-chip," IEEE Des. Test Comput., vol/issue: 31(5), pp. 55-65, 2014.

[21] A. Parini, et al., "BER evaluation of a passive SOI WDM router," IEEE Photon. Technol. Lett., vol/issue: 25(23), pp. 2285-2288, 2013.

[22] H. Gu, et al., "A Low-power Fat Tree-based Optical Network-on-Chip for Multiprocessor System-on-Chip," DATE, 2009.

[23] F. Xia, et al., "Ultra-compact high order ring resonator filters using submicron silicon photonic wires for on-chip optical interconnects," Optical Express, vol/issue: 15(19), pp. 11934-11941, 2007. 